\title{
STEMTooL: An Open Source Python Toolkit for Analyzing Electron Microscopy Datasets
}

\author{
Debangshu Mukherjee and Raymond Unocic \\ Oak Ridge National Laboratory, Oak Ridge, Tennessee, United States
}

The advent of aberration corrected electron optics and next generation detectors has opened a veritable flood gate of data that the modern electron microscope generates. This is varied across modalities - such as atomic resolution STEM imaging, EELS and EDS spectrum imaging or 4D-STEM datasets (where the electron diffraction pattern is collected at every scan position) [1]. Techniques such as 4D-STEM can be realistically thought of as an umbrella technique that includes strain mapping (from measurements of diffraction disk locations) [2,3], mapping of polar distortions (through comparison of higher order disk intensities) [4], differential phase contrast microscopy (through the measurement of the center of mass of the electron beam every scan position to calculate electromagnetic fields) [5], or ptychography (where the oversampled diffraction patterns are used to calculate the transfer function the electron beam experiences, which can be subsequently used for correction of lens aberrations and thus super-resolution imaging) [6]. Thus, there is a critical need to develop a complete toolkit for analyzing these complex datasets.

Two recent approaches have been hyperspy and py4DSTEM, which have respectively focused on analyzing EELS and 4D-STEM datasets [7,8]. Here we present stemtools, a Python module with opensource MIT licensing that can be used for analyzing 2D ADF-STEM images and 4D-STEM datasets [9].

Fig. 1(a) demonstrates a conventional atomic resolution ADF-STEM imaging of $\mathrm{PdCoO}_{2}$, where the atom positions are fitted and refined for sub-pixel precision, allowing for high precision measurements of strain, distortions or ferroelectric displacements from ADF-STEM datasets. This functionality is available through the afit submodule in stemtools. Along with conventional ADF-STEM, the Ronchigram can also be collected at every scan position, whose sum (a synthetic BF-STEM image) is shown in Fig. 1(b) for a monolayer and bilayer MoSe2 sample. Fig. 1(c) demonstrates the shift in the center of mass at every scan position, with Fig. 1(d) showing a zoomed in section where the arrows demonstrate the direction of the shift. The center of mass shifts could also be used to generate a "charge" map by differentiating the shifts, with Fig. 1(e) showing such a map, demonstrating how the bilayer region of $\mathrm{MoSe}_{2}$ shows a higher "charge" as compared to the monolayer region. This entire workflow is implemented in the dpc submodule of stemtools, which can be used to perform both atomic resolution and nanobeam electron diffraction quantification of electric fields.

Another widely applicable use case for 4D-STEM is nanobeam electron diffraction which can be used to quantify strain with sub-picometer precision across large fields of view [3]. The nbed submodule of stemtools allows for such strain comparison, with Fig. 2(a) demonstrating a synthetic BF-STEM image of a cluster of nanoparticles obtained by summing the undiffracted 000 electron beam. The nbed submodule allows for preconditioning the datasets through Sobel filtering of the log of the patterns to increase the strain quantification precision (Fig. 2(b)), with the filtering tools available in the utils submodule [10]. For strain quantification in the nanobeam electron diffraction mode, the electron beam convergence angle is reduced from 30mrad (used for atomic resolution imaging) to 5mrad, which generates a larger beam in real space, and prevents the individual higher order diffraction disks from overlapping, thereby allowing each diffraction spot to be located separately. The sub-pixel precision location of diffraction disks is performed by cross correlating a template with the preconditioned CBED pattern, and subsequently fitting the correlation peaks with 2D Gaussian functions [2], available in the 
utils submodule. The fitted disk positions can be used to calculate strain maps for an individual nanoparticle, as demonstrated in Fig. 2(c) - 2(f).

We have thus demonstrated a open-source Python based toolkit, that allows researchers to analyze both conventional STEM and 4D-STEM datasets. Further efforts are underway to package the toolkit as a selfcontained conda or pip installable machine independent distribution [11].

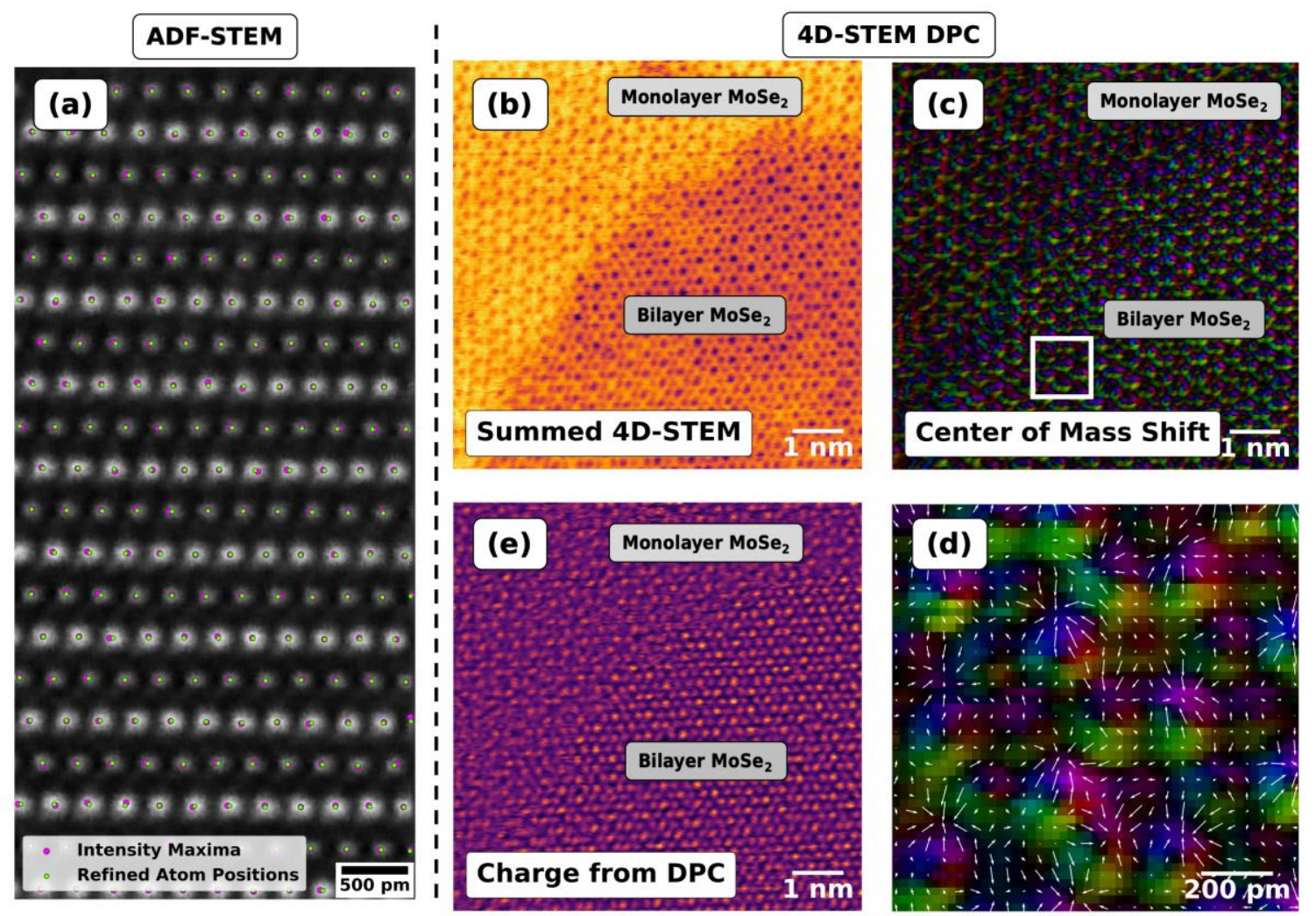

Figure 1. (a) Identifying atom positions in an ADF-STEM image of PdCoO2 through peak fitting. Combining atomic resolution STEM with DPC, (b) shows a synthetic BF-STEM image of bi-layer and monolayer MoSe 2 obtained by summing the Ronchigram at every scan position, while (c) shows the center of mass shifts in the simultaneously collected Ronchigram images, with (d) demonstrating the displacements in a zoomed out section of Fig. 1(c) marked by the white box. (e) demonstrates the calculated charge from the center of mass shifts. 

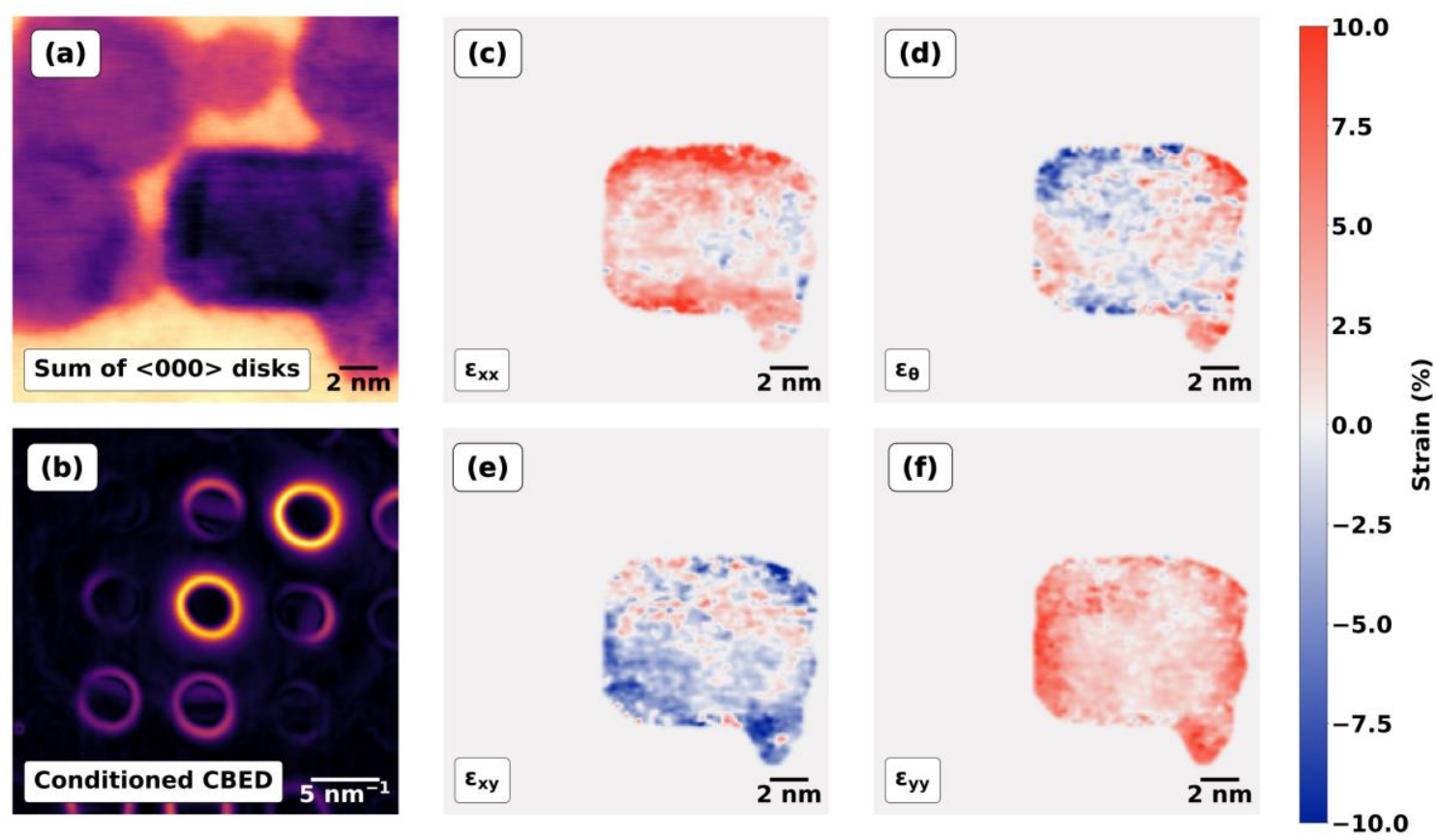

Figure 2. (a) Sum of the central disks of several nanoparticles, with a smaller convergence angle (5mrad) for strain mapping. (b) Preconditioned diffraction patterns collected with a smaller convergence angle to ensure no diffraction disks overlap. (c) - (f) $\varepsilon x x, \varepsilon x y, \varepsilon \theta$ and $\varepsilon y y$ strain maps calculated by comparing the higher order diffraction disk positions with the central undiffracted () disk.

\section{References}

[1] Ophus, C. (2019). Microscopy and Microanalysis, 25(3), pp.563-582

[2] Pekin, T.C., et. al. (2017.) Ultramicroscopy, 176, pp.170-176.

[3] Han, Y., et. al. (2018). Nano letters, 18(6), pp.3746-3751

[4] Yadav, A.K., et. al. (2019). Nature, 565(7740), pp.468-471.

[5] Müller, K., et. al. (2014). Nature communications, 5(1), pp.1-8.

[6] Jiang, Y., et. al. (2018). Nature, 559(7714), pp.343-349.

[7] de la Peña, F., et. al. (2017). Microscopy and Microanalysis, 23(S1), pp.214-215.

[8] Savitzky, B.H., et. al. (2019). Microscopy and Microanalysis, 25(S2), pp.124-125.

[9] Mukherjee, D. (2020). http://doi.org/10.5281/zenodo.3686169.

[10] Mukherjee, D., et. al. (2020). arXiv preprint arXiv:2001.01010.

[11] Research supported by ORNL's Laboratory Directed Research and Development Program, which is managed by UT-Battelle, LLC for the U.S. Department of Energy (DOE). Electron microscopy was conducted as part of a user proposal at Oak Ridge National Laboratory's Center for Nanophase Materials Sciences (CNMS), which is a U.S. DOE Office of Science User Facility. 